\title{
The Partonic Nature of Instantons
}

\section{David Tong}




\section{Happy Birthday Misha!!}




\section{What are Instantons Made of?}

- Instantons have a number of collective coordinates:

- SU(N) Yang-Mills

- 4 translations, 1 scale size, $4 \mathrm{~N}-5$ orientation

- $4 \mathrm{~N}$ in total

- CPN Sigma-Model

- 2 translations, 1 scale size, $2 \mathrm{~N}-3$ orientations

- $2 \mathrm{~N}$ in total

- An old idea: Instantons are composed of $\mathrm{N}$ partons 


\section{The Partonic Nature of Instantons}

- The conjecture of partons usually framed for

- $d=3+1$ dimensions (for Yang-Mills)

- $\quad \mathrm{d}=1+1$ dimensions (for sigma-models)

- Here we revisit this idea in context of

- $\mathrm{d}=4+1$ dimensions (for Yang-Mills)

a $\quad \mathrm{d}=2+1$ dimensions (for sigma-models)

- Theories are non-renormalizable (effective theories)

- Instantons are particle-like excitations

- Is the single instanton a multi-particle state?

a ...and how can we tell? 


\section{5d Yang-Mills}

- There exists a UV completion of gauge theories in $d=4+1$ dimensions

- (at least with supersymmetry)

- ...but we don't know much about it

- With $\mathrm{N}=2$ supersymmetry, the UV completion is the $(2,0)$ theory in $\mathrm{d}=5+1$ dimensions

- The UV completion has $\mathrm{N}^{3}$ degrees of freedom. Klebanov and Tseytlin ‘96 


\section{The Instanton}

- The instanton is the $\mathrm{KK}$ mode from the sixth dimension

$$
M_{\mathrm{inst}}=\frac{8 \pi^{2}}{e^{2}}=\frac{1}{R}
$$

- Proposal: The instanton is an N-particle state

- The $\mathrm{N}$ partons inside the instanton are the remnant of the UV degrees of freedom which comprise the $(2,0)$ theory. 


\section{Circumstantial Evidence}

- Turn up the heat and look at cross-over in free-energy

$$
F \sim N^{2} T^{5} \longrightarrow R N^{3} T^{6}
$$

- The transition happens at the temperature

$$
T \sim \frac{1}{N R} \sim \frac{1}{e^{2} N}
$$

- This had to be the case: this is where the $5 d$ theory is strongly coupled, so this is where we need new UV degrees of freedom. 


\section{More Circumstantial Evidence}

- Anomaly coefficient for $G=A D E$ is conjectured to be

dual coxeter number

$=$ number of instanton moduli

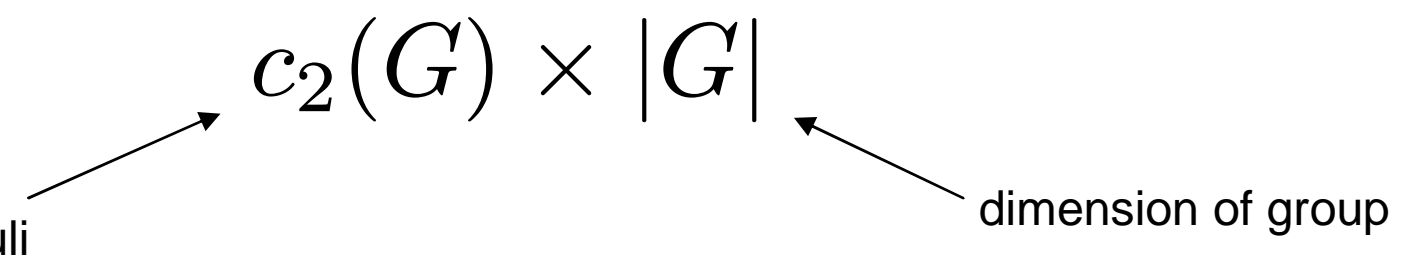

- Are the partons in the adjoint of $\mathrm{G}$ ?

a What is the mechanism that confines them?

- Quantizing the scale size of the instanton gives a continuous spectrum...natural for a multi-particle state.

- But the moduli space is not that of $\mathrm{N}$ free objects...does this contain clues about confining mechanism? 


\section{A Toy Model}

- These questions are difficult to answer in case of Yang-Mills

- The $\mathrm{CP}^{\mathrm{N}}$ sigma-model provides (as always!) a nice toy-model where we can see how some of these issues are resolved. 


\section{A Toy Model}

- Our toy will be in $\mathrm{d}=2+1$ dimensions

- It is a gauge theory with $\mathrm{N}=4$ supersymmetry

a Vector multiplet: $V=\left(A_{\mu}, \phi_{i}\right.$, fermions $) \quad i=1,2,3$

口 Hypermultiplet: $Q=(q, \tilde{q}$, fermions $)$

- $U(1)^{N}+\mathrm{N}$ hypermultiplets

- gauge coupling $=g^{2}$

- mass of hypers $=\mathrm{m}$

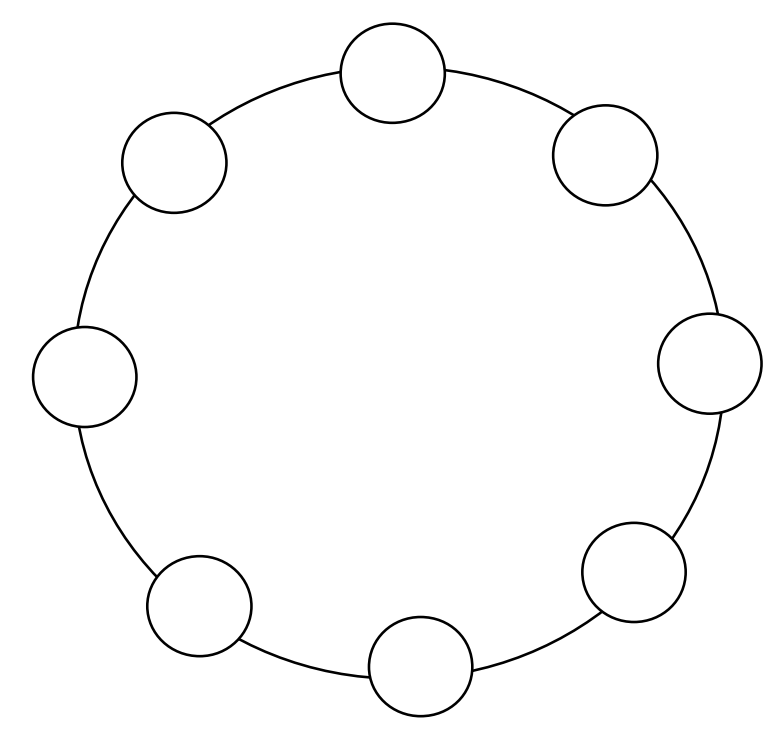




\section{The Low-Energy Dynamics}

- The hypermultiplets have physical mass

$$
M^{a}=\left(\phi^{a}-\phi^{a+1}+m\right)
$$

vevs of vector multiplets

- At low energies, we want an effective action for the massless vector multiplets

$$
\phi_{i}^{a} \text { and } F_{\mu \nu}^{a}=g^{2} \epsilon_{\mu \nu \rho} \partial_{\rho} \sigma^{a}
$$

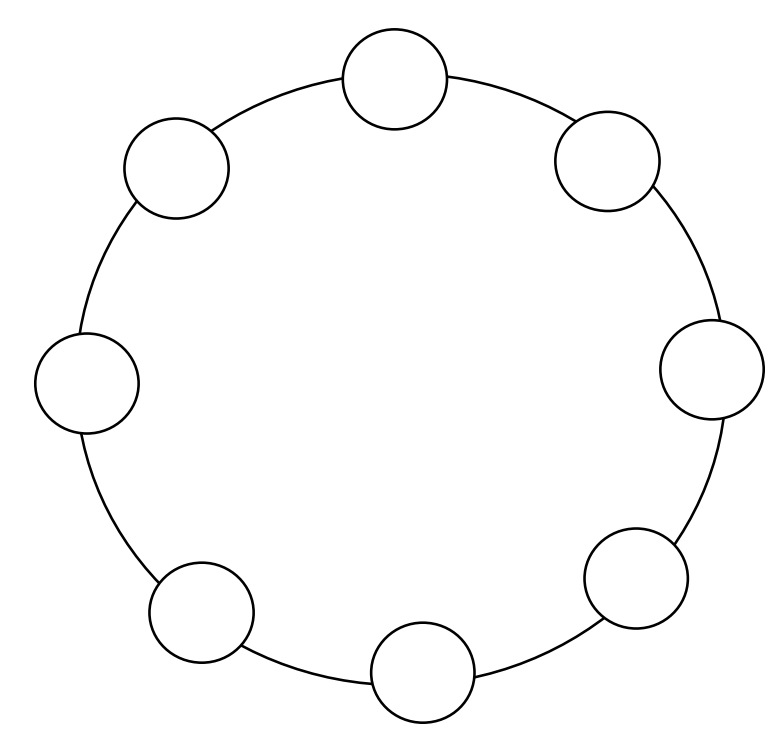




\section{Low-Energy Dynamics}

- We integrate out the hypermultiplets

- This induces interactions for vector multiplets

- The low-energy dynamics is a sigma-model with target space

$$
\mathbf{R}^{4} \times T^{\star} \mathbf{C P}^{N-1}
$$

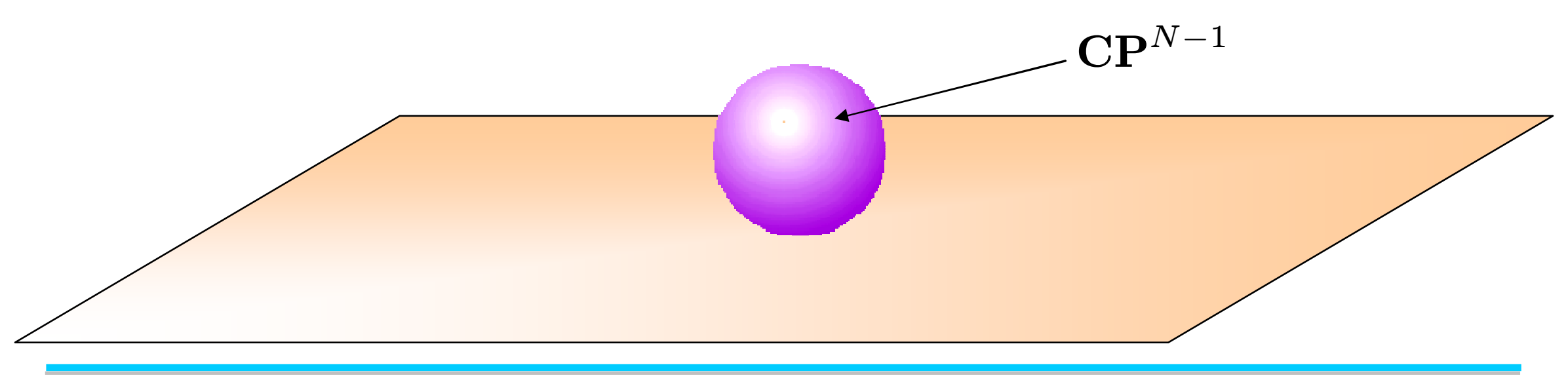




\section{An Example: $\mathbf{C P}^{\mathbf{1}}$}

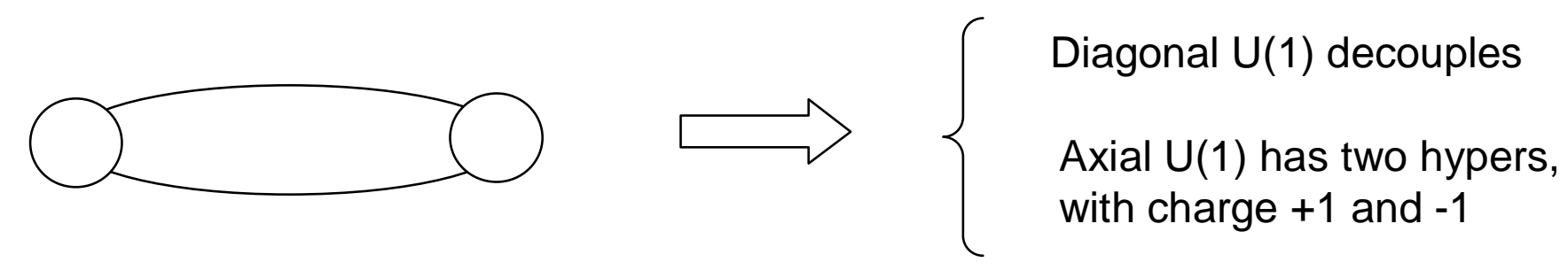

Integrate out hypers: $\quad \mathcal{L}_{\text {eff }}=\frac{1}{g_{\text {eff }}^{2}}(\partial \phi)^{2}+g_{\text {eff }}^{2}(\partial \sigma)^{2}$

$$
\frac{1}{g_{\mathrm{eff}}^{2}}=\frac{1}{g^{2}}+\frac{1}{m-\phi}+\frac{1}{m+\phi}
$$
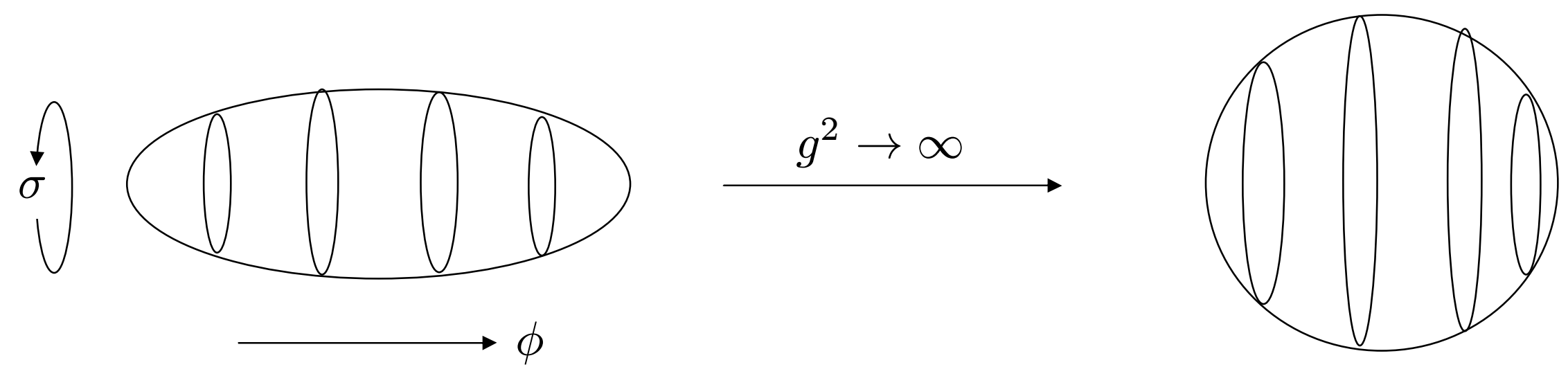


\section{The Soliton}

- The low-energy theory has a soliton: it is a sigma-model lump

- 2 translation modes

- 1 scale size

- $2 \mathrm{~N}-3$ orientation modes

$$
\partial_{\mu} \phi=g_{\text {eff }}^{2}(\phi) \epsilon_{\mu \nu} \partial^{\nu} \sigma
$$

- What is this soliton in the microsopic theory?
- It is BPS
- Mass $=\mathrm{Nm}$

- It is an N-particle state.

- The soliton is made of the objects that we thought we'd integrated out!

$$
Q_{1} Q_{2} Q_{3} \ldots Q_{N}
$$

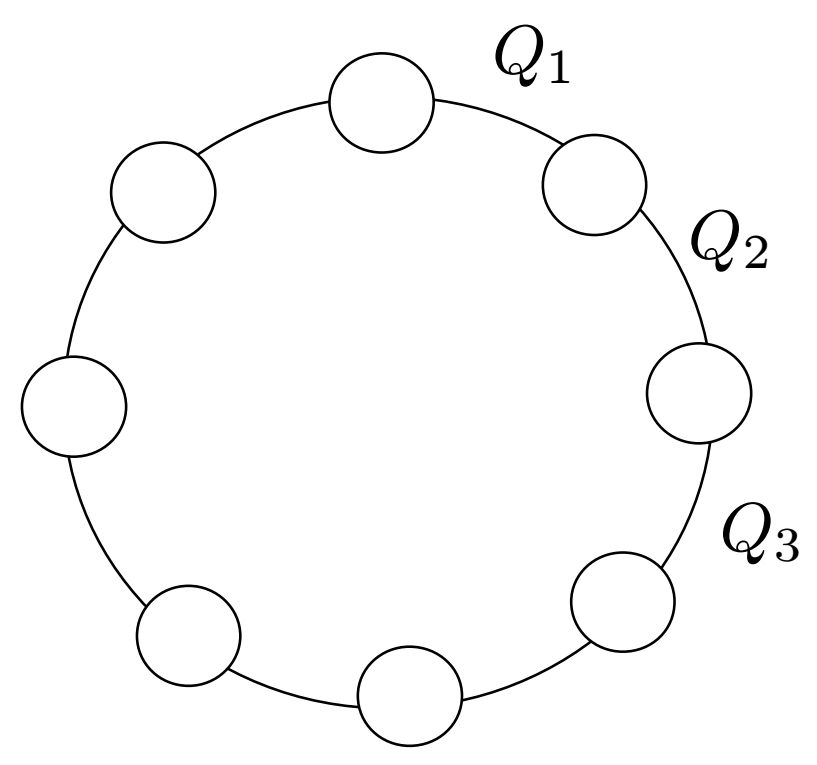




\section{Reconstructing the UV Physics}

- Question: Suppose we just have access to the IR physics on the Coulomb branch. What does the soliton tell us about the UV completion?

- Answer: Pretty much everything! 


\section{Seeing the Partons}

- We get the round metric on $\mathbf{C P}^{N-1}$ only in the limit $g^{2} \rightarrow \infty$

- If we study solitons on the squashed target space, the solitons dramatically reveal themselves. Here's a soliton on $\mathbf{C P}^{1}$
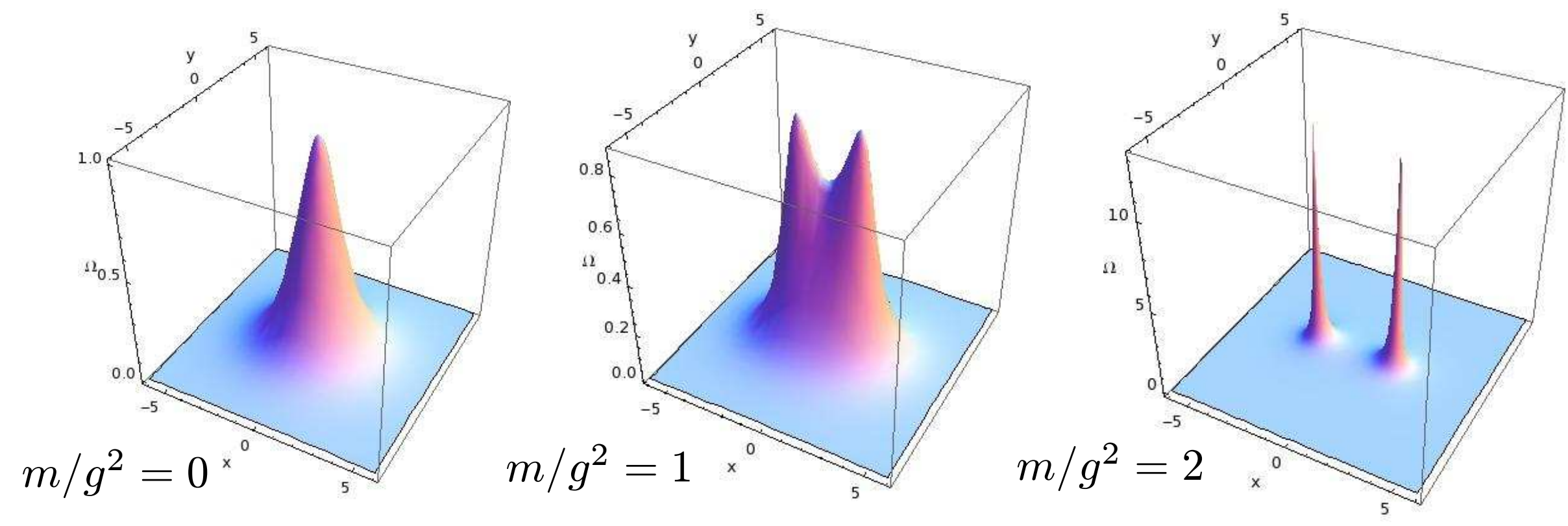


\section{Seeing the Partons}

- Here's a single soliton on a squashed $\mathbf{C P}^{2}$
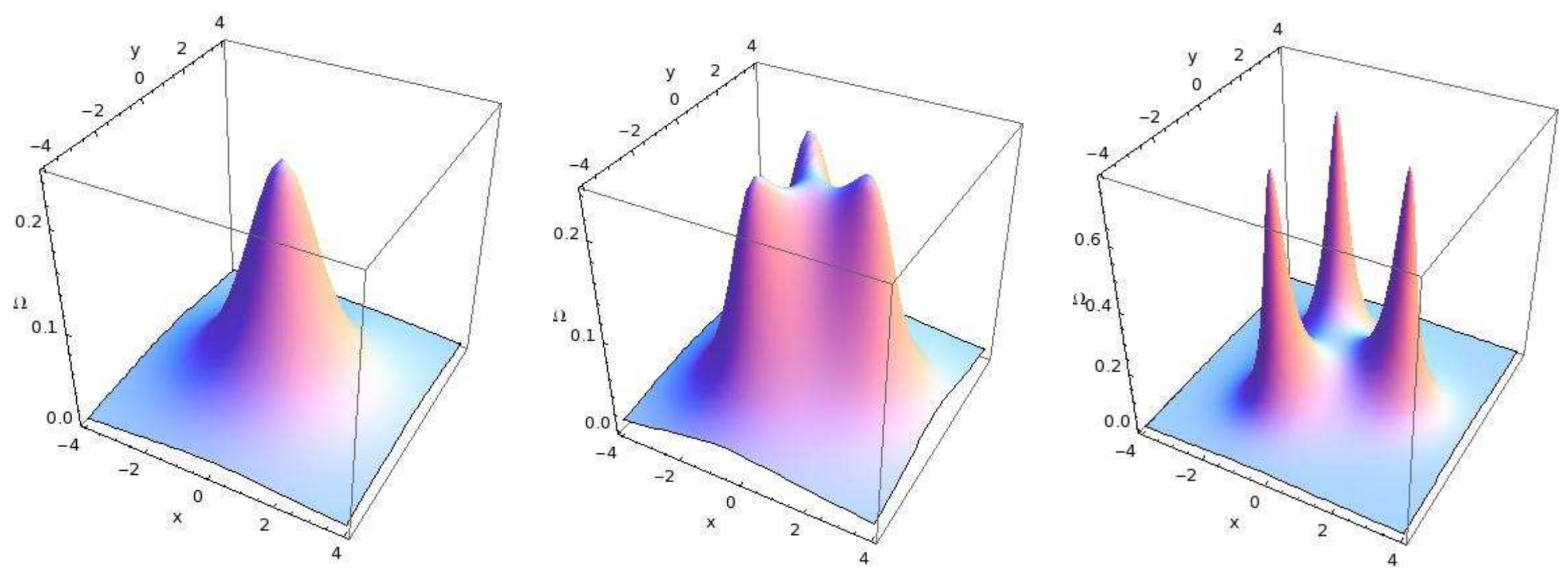


\section{Changing the Orientation}

- Keep the "scale size" of the lump fixed

- Change the "orientation modes"

- Watch the partons move
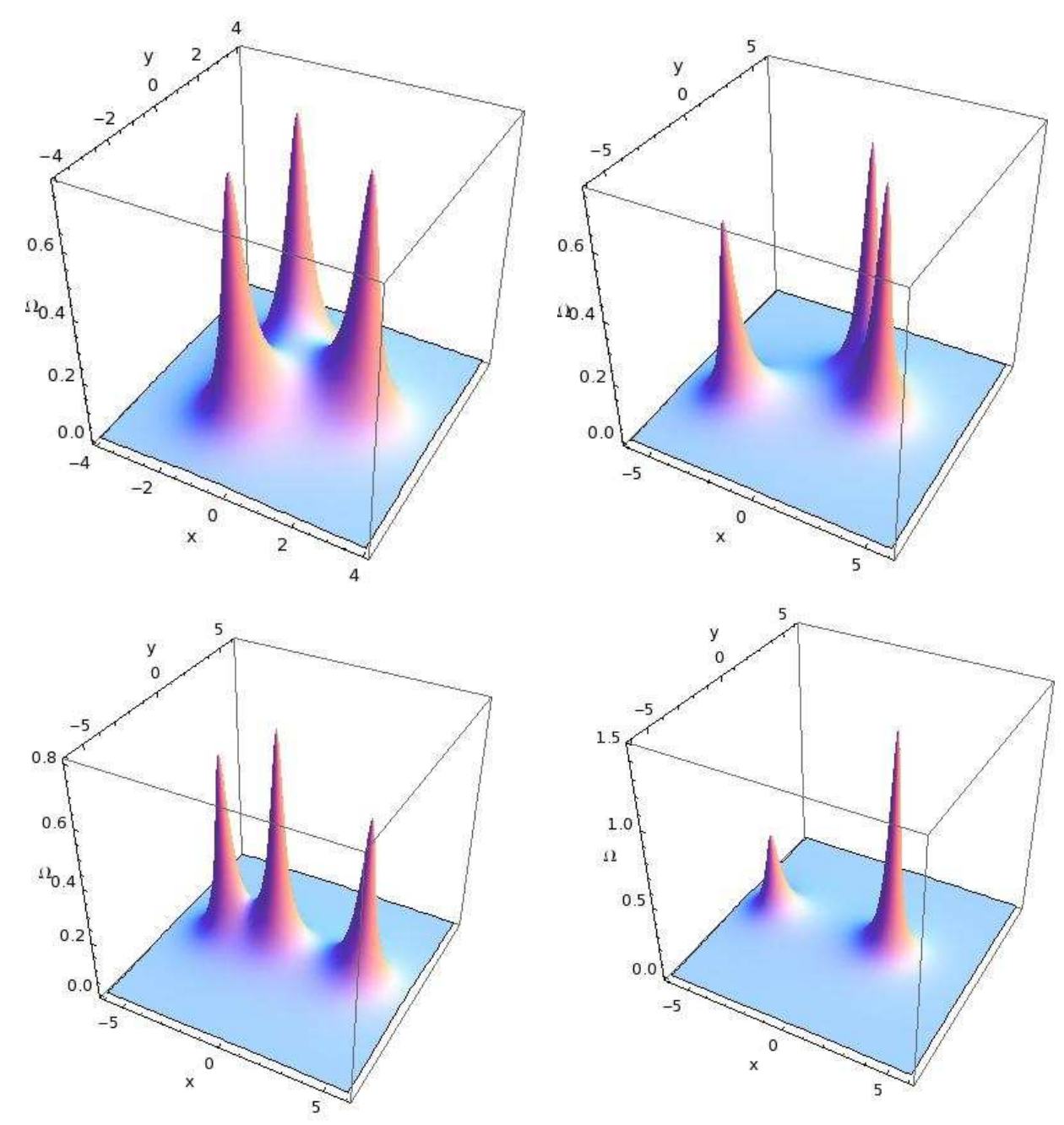


\section{Confinement of Partons}

- Why does the low-energy theory only include the $\mathrm{N}$-particle state?

$$
Q_{1} Q_{2} \ldots Q_{N}
$$

- Answer from microscopic theory: logarithmic confinement

- In $\mathrm{d}=2+1$ dimensions, electric charges have $E \sim \frac{1}{r}$

- This gives log divergent mass

- Only gauge singlet states have finite mass

- This log divergence re-appears when partons move.

- Seen in IR theory as a log divergence in moduli space metric. Only modes which don't

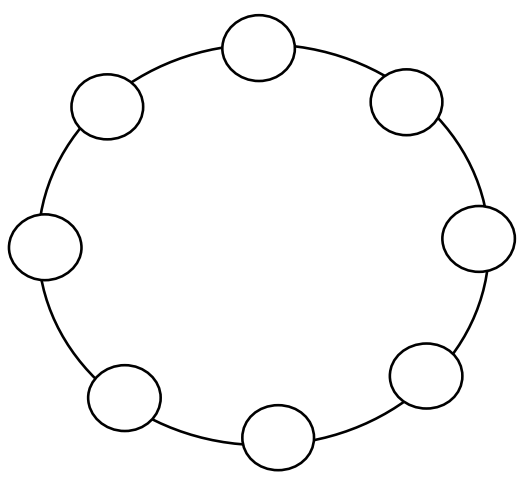
change dipole moments have finite norm 


\section{Parton Quantum Numbers}

- Can we reconstruct the quantum numbers of the partons? 口 In other words, can we reconstruct the full UV theory?

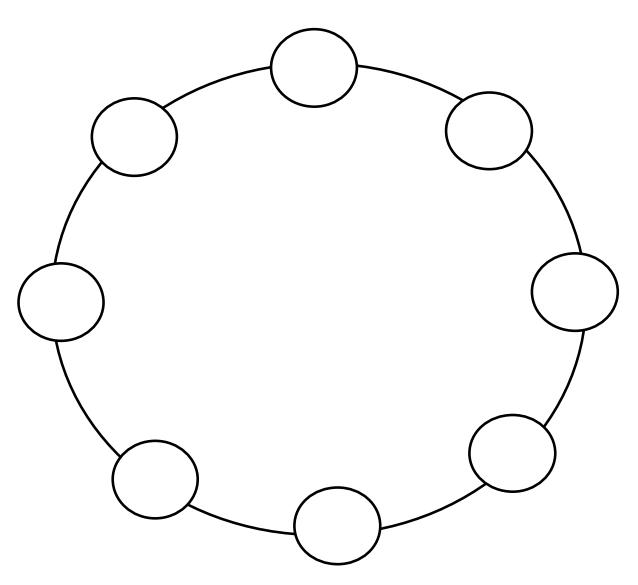




\section{Dual Bogomolnyi Equations}

- The Bogomonyi equations for $\mathbf{C P}^{\mathbf{1}}$ are

$$
\partial_{\mu} \phi=g_{\text {eff }}^{2}(\phi) \epsilon_{0 \mu \nu} \partial^{\nu} \sigma
$$

- They have a moduli space of solutions: dimension $2 \mathrm{kN}$ for $\mathrm{k}$ soliton sector

- Can rewrite this equation in dual variables $F_{\mu \nu}=g_{\mathrm{eff}}^{2}(\phi) \epsilon_{\mu \nu \rho} \partial_{\rho} \sigma$

$$
F_{0 \mu}=\partial_{\nu} \phi
$$




\section{Dual Bogomolnyi Equations}

$$
F_{0 \mu}=\partial_{\nu} \phi
$$

- The dual Bogomolnyi equations have no smooth solutions

- But we can reproduce the exact soliton solution if we introduce electric sources

$$
\partial_{\mu}\left(\frac{1}{g_{\text {eff }}^{2}} F_{0 \mu}\right)=\sum_{n=1}^{k} \delta\left(z-z_{n}^{+}\right)-\delta\left(z-z_{n}^{-}\right)
$$

In soliton description, these are collective coordinates. Here, they are sources. 


\section{Dual Bogomolnyi Equation}

- The dual formulation of the Bogomolnyi equation provides an explicit map between a soliton and fundamental fields

- It also works for $\mathrm{CP}^{\mathrm{N}-1}$

- Can reconstruct quantum numbers of partons which determines the UV microscopic theory

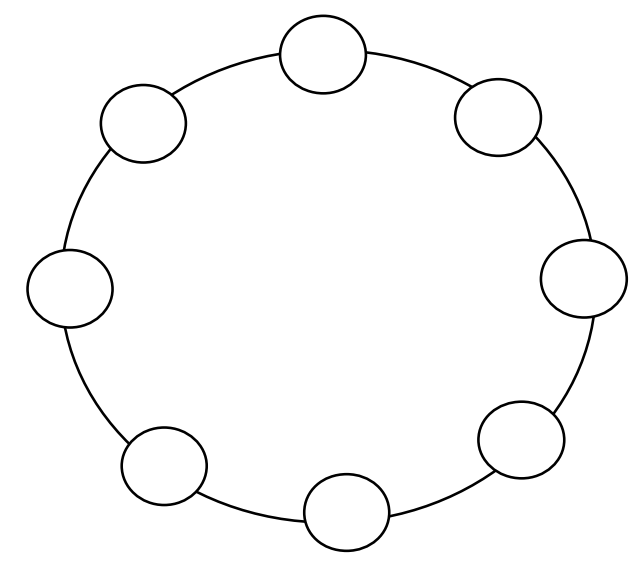




\section{Calorons: A Red Herring?}

- There is one way that instantons are known to split into $\mathrm{N}$ partons

- Calorons

- Put $d=4+1$ theory on circle. Add a Wilson line.

- Instantons $\longrightarrow \mathrm{N}$ monopole strings

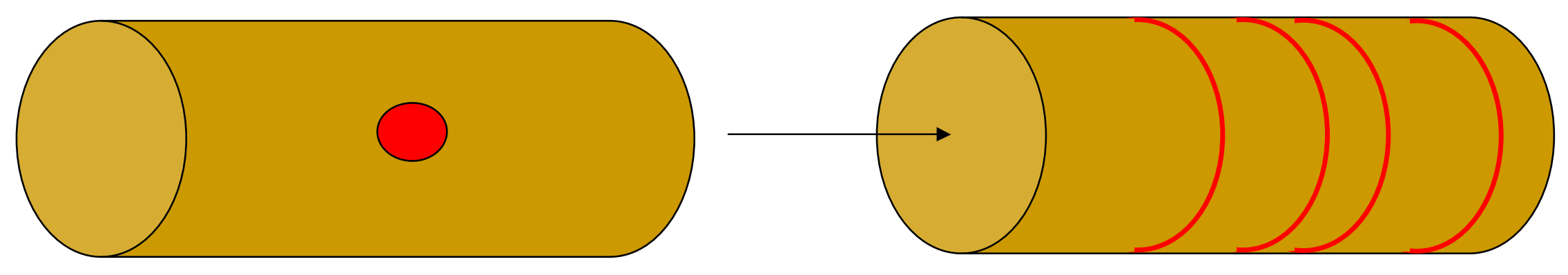

- Doesn't seem possible that this can happen in non-compact space

- Also happens for lumps in the toy model

a But the calorons have nothing to do with the true partons 


\section{Summary: Questions, not Answers}

- Toy model in $\mathrm{d}=2+1$

- Explicit demonstration that solitons can be thought of as multi-particle states

a A study of the soliton allows us to reconstruct the UV behaviour

- Real Interest: $d=4+1$ Yang-Mills

- Does the instanton solution hold clues about the constituents of the $(2,0)$ theory?

- What is the confinement mechanism?

- No hint of log confinement...partons are probably not merons 


\section{Happy Birthday Misha!!}

